\title{
Flip-Chip Bonding of VCSELs to Silicon Grating Couplers via SU8 Prisms Fabricated using Laser Ablation
}

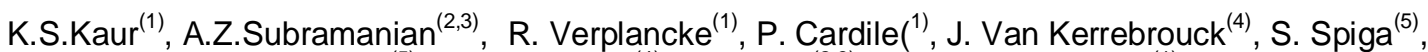 \\ R. Meyer ${ }^{(5)}$, J. Bauwelinck ${ }^{(4)}$, R. Baets ${ }^{(2,3)}$, G. Van Steenberge ${ }^{(1)}$ \\ (1) Centre for Microsystems Technology, imec/Ghent University, B-9052 Gent, Belgium \\ (Kaur.Kamalpreet@elis.ugent.be) \\ (2) Photonics Research Group, INTEC Department, Ghent University-imec, Ghent 9000, Belgium \\ (3) Centre for Nano- and Biophotonics, Ghent University, Ghent 9000, Belgium \\ (4) Deparment of Information Technology, Ghent University-iMinds-imec, B-9000 Ghent, Belgium \\ (5) Walter Schottky Institut, Technische Universität München, D-85748 Garching, Germany
}

\begin{abstract}
We successfully demonstrate for the first time, VCSEL-to-silicon grating coupler flip-chip assembly using SU8 prism fabricated by non-uniform laser ablation. Excess loss of $<1 \mathrm{~dB}$ and data transmission up to $20 \mathrm{~Gb} / \mathrm{s} @ 1550$ nm was achieved.
\end{abstract}

\section{Introduction}

In silicon photonics, the use of grating couplers (GCs) is a standard solution to connect on-chip circuitry to the outside world. However, the conventional GC designs require off-normal (typically 8-10 degrees) coupling angle so as to avoid second order Bragg reflections. This offnormal incidence condition makes the integration of light sources especially verticalcavity surface-emitting lasers (VCSELs) to silicon-on-insulator (SOI) chips a challenging task, as the back reflections not only lead to poor coupling efficiencies but are also detrimental for the VCSEL performance. Although complex designs of GCs adapted for perfect vertical coupling have been reported ${ }^{1}$, they are hard to implement. Another approach could be to use the standard GCs and flip-chip bond the VCSELs on top at an angle but that again is a non-trivial and non-standard technology. An alternative solution to this problem is by incorporating micro-optical elements on top of GCs as reported in [2]. In this paper, fiber-to-fiber transmission was demonstrated using a polymer (SU8 and PAK) wedge fabricated using focused-ion-beam (FIB) and imprinting lithography on top of the GCs to translate the vertical incidence into an off-normal incidence. However, FIB is inherently a timeintensive, expensive and non-flexible process. Finally, as the polymer wedge is protruding out on the SOI chip ${ }^{2}$ patterning the bonding pads and realizing VCSEL-to-SOI chip assembly using this approach can be quite challenging.

In order to circumvent the above mentioned issues, we present a laser-based, contactless and single-step process to fabricate polymer prisms on top of the GCs. It is a simple, fast, flexible and wafer-scalable process. In this paper, we demonstrate for the first time the integration of VCSEL chips to silicon GCs using SU8 prisms. The VCSELs were flip-chipped onto the SOI chips employing the laser-induced forward transfer (LIFT) - assisted thermocompression bonding ${ }^{3}$. We describe in this paper the technological approach and the measurement of coupling efficiency. We also report on the high speed data transmission experiments performed on the bonded assemblies.

\section{Flip-chip bonding of VCSEL to SOI}

The waveguides and GCs used for the experiments were fabricated using deep UV lithography in a CMOS environment via ePIXfab ${ }^{4}$. The waveguides were completely etched and were $220 \mathrm{~nm} \times 500 \mathrm{~nm}$ in dimensions. The GCs were shallow etched by $70 \mathrm{~nm}$ at the input and output end of the waveguide. The entire structure was cladded by $\mathrm{SiO}_{2}$ on top for reduced losses.

The various steps involved in the process flow to realize the VCSEL to SOI assembly are depicted in Fig. 1. First of all the insertion loss of the SOI chips (GC-WG-GC) was measured prior to the prism fabrication to get the reference loss value. The GCs used for the experiments were designed for $10^{\circ}$ operation in the $\mathrm{C}$-band for TE polarization mode. For the measurements light from a tunable laser source was coupled into the input GC at an angle of $10^{\circ}$ using a SMF (single mode fiber) and was collected after propagation in the SOl waveguide from the output GC using another SMF at an angle of $10^{\circ}$. A $25 \mu \mathrm{m}$ thick blanket layer of SU8 was then spin coated on top of the SOI chip, followed by soft bake (1 $\mathrm{min}$ @ 65 deg, 10 min @ 95 deg), flood exposure (> $300 \mathrm{~mJ} / \mathrm{cm}^{2}$ ) and hard bake (1hr@ @ $\left.150 \mathrm{deg}\right)$ steps to fully cure the SU8 layer. Next, the VCSEL bonding pads ( $\mathrm{Ti} / \mathrm{Ni} / \mathrm{Au})$ were defined on 
top of the SU8 layer using image reversal lithography.
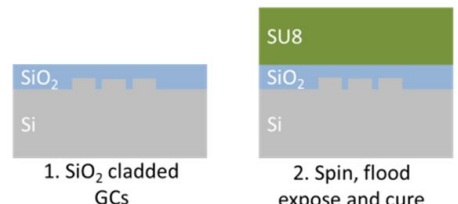

2. Spin, flood
expose and cure SU-8 layer

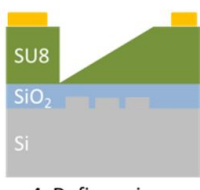

4. Define prism using laser ablation
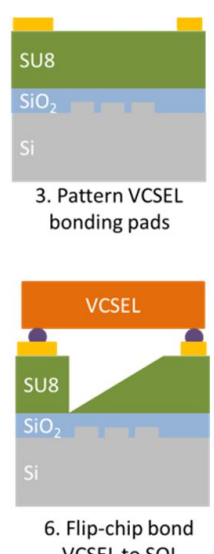

VCSEL to SOI
Vlip-chip bond
Pattern VCSEL

Fig. 1: Schematic depicting the process flow for VCSEL-toSOI integration (not drawn to scale).

The next step was the fabrication of SU8 prisms with an angle of $24^{\circ}$ on top of the GCs using non-uniform laser ablation ${ }^{5}$. The optimum value for the prism angle was calculated using Snell's law for a normal incidence on prism face such that it resulted into a $10^{\circ}$ incidence angle onto the GCs. Laser pulses from an excimer laser (KrF, operating wavelength $248 \mathrm{~nm}$, pulse duration 5-8 ns, pulse energy $570 \mu \mathrm{J}$ ) were first passed through a triangular mask $(1000 \times 610 \mu \mathrm{m})$ and a demagnified image (factor of 12) of the mask was projected onto the SU8 coated sample. The sample was then scanned in a direction perpendicular to the incoming laser beam at a speed of $157 \mu \mathrm{m} / \mathrm{s}$ at $100 \mathrm{~Hz}$ repetition rate to ablate a $\sim 22 \mu \mathrm{m}$ deep prism with $24^{\circ}$ angle. The simplicity of this technique lies in the fact that the prism angle can be tuned just by adjusting the laser energy and sample scan speed. Microbumps of indium metal with $\sim 20 \mu \mathrm{m}$ diameter and $7 \mu \mathrm{m}$ thickness were then printed on the bonding pads employing the LIFT technique. The details about this technique can be found in [3]. A Timebandwidth Duetto laser source (355 $\mathrm{nm}, 12 \mathrm{ps)}$ and a transfer fluence of $300 \mathrm{~mJ} / \mathrm{cm}^{2}$ was used for the LIFT experiments. The final step in the assembly was to flip-chip bond the VCSEL chips onto the GCs with SU8 prism fabricated on top. The $1550 \mathrm{~nm} n$-InP VCSELs used in these experiments were based upon a short-cavity design and had three compressively strained AlGalnAs quantum wells ${ }^{6}$. A semiautomatic flip-chip bonder from Tresky was used for thermocompression bonding of the VCSELs to the SOI chips. A mechanical and electrical interconnection between the VCSEL and the SOI chip was established via molten indium bumps ${ }^{3}$.

\section{Characterization of the bonded assembly}

The samples were measured after each processing step (1-6 in Fig. 1) to evaluate the excess loss (if any) introduced by the processing to ensure an optimum coupling efficiency from the GCs. The loss values measured at various stages are listed in Tab. 1. Prior to VCSEL bonding fiber to fiber transmission measurements were performed. Till step 3 both the input and output fibers were tilted at $10^{\circ}$ angle from the normal to GC, while from step 4 onwards, the input fiber was positioned along the normal $\left(0^{\circ}\right)$ and the prism refracted the incident light to an optimum $10^{\circ}$ onto the GCs. No significant shift in the GC efficiency was recorded at any stage of process flow indicating there was no excess loss due to any step and the process was optimum. The fiber transmission curves recorded before and after prism fabrication for one of the samples is shown in Fig. 2.

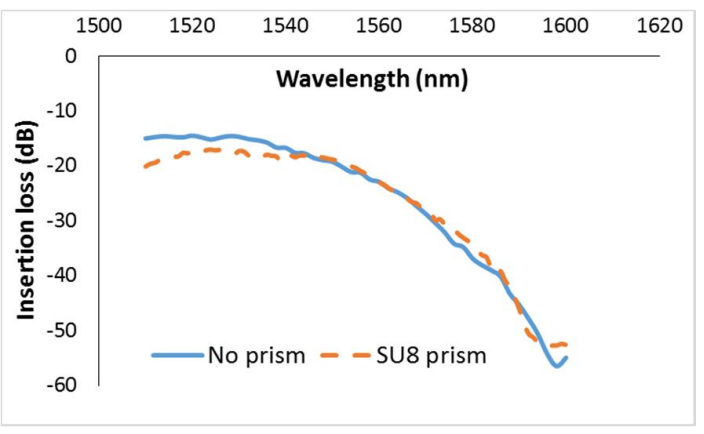

Fig. 2: Transmission curves recorded before and after prism fabrication onto GCs.

A typical bonded assembly comprising of bonding pads, prism, SOI and VCSEL is shown in Fig. 3. Before bonding, the VCSEL chips were characterized and they typically emitted $-4 \quad \mathrm{dBm} @ 5 \mathrm{~mA}$. For the DC measurements a driving current of $5 \mathrm{~mA}$ was applied to the bonded VCSELs. The emitted power was coupled into the SOI waveguide via SU8 prism and GC combination. After propagating in the waveguide the power was collected at the output GC by a SMF at $10^{\circ}$ and was recorded by a power meter. Few devices were tested and for the best case the transmitted power collected at the output was up to $-21.7 \mathrm{dBm} @ 1547 \mathrm{~nm}$ (peak wavelength of VCSEL) for a driving current of $5 \mathrm{~mA}$. This resulted in a total excess loss of $<1 \mathrm{~dB}$ introduced by the whole assembly process as shown in Tab. 1. The main contributing factor for the excess loss is attributed to the misalignment during bonding as the flip-chip process involved passive alignment of the VCSEL and SOI chip. 
Tab. 1: Loss values measured at various stages of the VCSEL-to-SOI integration process.

\begin{tabular}{|c|c|c|c|c|}
\hline Sample ID & $\begin{array}{c}\text { Fiber-to-waveguide coupling } \\
\text { efficiency without prism } \\
\left(10^{\circ}, \mathbf{1 5 5 0} \mathrm{nm}\right) \\
(\mathrm{dB})\end{array}$ & $\begin{array}{c}\text { Fiber-to-waveguide } \\
\text { coupling efficiency with } \\
\text { prism } \\
\left(0^{\circ}, \begin{array}{c}1550 \mathrm{~nm}) \\
(\mathrm{dB})\end{array}\right.\end{array}$ & $\begin{array}{c}\text { VCSEL-to- } \\
\text { waveguide } \\
\text { coupling } \\
\text { efficiency } \\
(@ 5 \mathrm{~mA}) \\
(\mathrm{dB})\end{array}$ & $\begin{array}{c}\text { Total excess loss } \\
\text { (prism+bonding) } \\
\text { (dB) }\end{array}$ \\
\hline S1 & -18.5 & -19 & -20 & -1.5 \\
\hline S2 & -17 & -17.5 & -17.7 & -0.7 \\
\hline
\end{tabular}

To further check the reliability and the performance of the process, non-return-to-zero (NRZ) transmission experiments till $20 \mathrm{~Gb} / \mathrm{s}$ were performed on the bonded assemblies by direct modulation of the VCSELs. Clear and wide open eye patterns for 10 and $15 \mathrm{~Gb} / \mathrm{s}$ were recorded representing an error free transmission as shown in Fig. 4. At $20 \mathrm{~Gb} / \mathrm{s}$, the bandwidth limitation of the set-up started to appear.

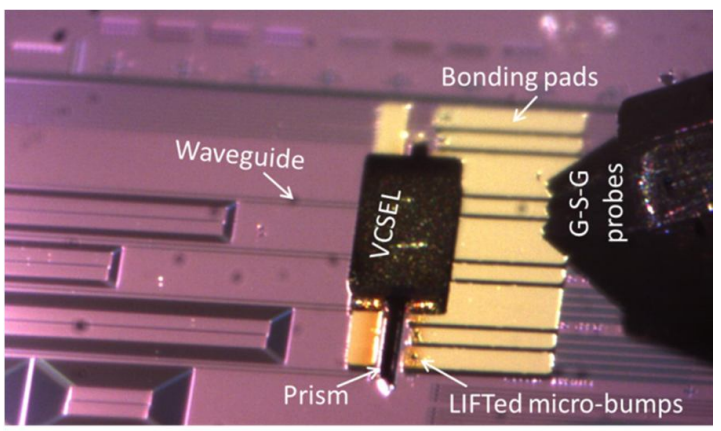

Fig. 3: Typical bonded assembly of VCSEL on top of SOI chip.

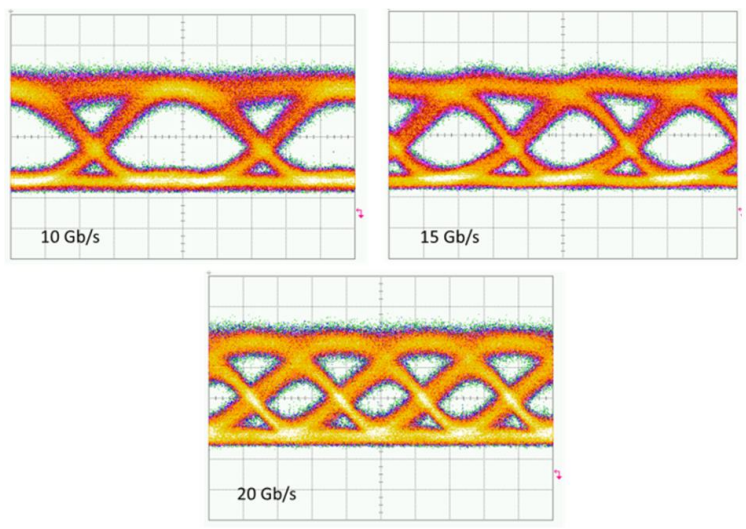

Fig. 4: Eye diagrams recorded for the bonded VCSEL assemblies at 10,15 and $20 \mathrm{~Gb} / \mathrm{s}$.

\section{Conclusions}

To conclude, flip-chip bonding of VCSEL chips to SOI GCs using SU8 prisms was successfully demonstrated in this paper. The step-by-step process for the SU8 prism fabrication on top of GCs employing non-uniform laser ablation followed by LIFT assisted thermocompression flip-chip bonding of VCSEL chips was presented. An excess loss of $<1 \mathrm{~dB} @ 1550 \mathrm{~nm}$ was measured for the bonded assemblies. NRZ measurements showed clear open eyes recorded till $20 \mathrm{~Gb} / \mathrm{s}$ for the bonded samples thereby confirming the suitability of this approach for high speed data transmissions. The simplicity, cost-effectiveness, flexibility and wafer scalability makes this method an attractive solution for VCSEL-to-SOI integration.

\section{Acknowledgements}

This work was carried out in the framework of the project MIRAGE (FP7-ICT-2011-8ICT) funded by the European Commission within the FP7 program. The authors would like thank Steven Verstuyft from PRG for his help with the evaporation step.

\section{References}

[1] G. Roelkens et al., "High efficiency grating coupler between silicon-on-insulator waveguides and perfectly vertical optical fibers," Opt. Lett. Vol. 32, no. 11, p. 1495 (2007).

[2] J. Schrauwen et al., "Polymer wedge for perfectly vertical light coupling to silicon," Proc. SPIE, 72180B, San Jose (2009).

[3] K. S. Kaur et al., "Flip-chip bonding of vertical-cavity surface-emitting lasers using laser-induced forward transfer," Appl. Phys. Lett., Vol. 104, no. 6, p. 061102 (2014).

[4] http://www.epixfab.eu

[5] J.E.A Pedder et al., "Pulsed laser ablation for volume micro-optical arrays on large area substrates," Proc. SPIE, , 64620W, San Jose (2007).

[6] M. Müller, et al,"1550-nm high-speed short-cavity VCSELs, IEEE J. Sel. Topics Quantum Electron., Vol.17, p.1158, (2011). 